\title{
In-situ Synthesis of PtSn/C Fuel Cell Nanocatalysts in an Aberration-Corrected STEM/TEM
}

\author{
Jingyue Liu,* Janet Braddock-Wilking* and Lawrence F. Allard**
}

* Center for Nanoscience and Department of Chemistry and Biochemistry, University of MissouriSt. Louis, One University Boulevard, St. Louis, Missouri 63121 (liuj@umsl.edu)

** Materials Science and Technology Div., Oak Ridge National Laboratory, Oak Ridge, TN 37831

The decreasing amount of fossil fuels and the recent concerns about global warming have been driving forces for developing alternative and renewable energy sources such as biomass-derived liquid fuels. The PtSn/C nanocatalyst is now considered as the most effective catalyst for the anode performance of the direct ethanol fuel cell [1]. The optimum Pt/Sn ratio and their degree of alloying, however, still remain controversial; furthermore, the catalyst preparation procedure can significantly affect the catalyst's nature and performance [2-3]. To better understand the formation processes of the PtSn alloy nanoparticles on an atomic scale we studied the in-situ synthesis processes of $\mathrm{PtSn} / \mathrm{C}$ alloy nanocatalysts inside a JEOL 2200FS aberration-corrected electron microscope.

Molecules of the complex $\left(\mathrm{Ph}_{3} \mathrm{P}\right)_{2}(\mathrm{H}) \mathrm{Pt}\left(\mu-\mathrm{SnPh}_{2}\right)\left(\mu-\eta^{2}-\mathrm{H}-\mathrm{SnPh}_{2}\right) \mathrm{Pt}\left(\mathrm{PPh}_{3}\right)$ [4] were dispersed onto Vulcan XC-72 carbon black powders via an impregnation method. The precursor materials were dried under inert gas environment and then were reduced inside the JEOL 2200FS instrument, which is equipped with a CEOS GmbH. aberration corrector that provides a nominal image resolution of $0.07 \mathrm{~nm}$. Catalyst precursor materials were heated in-situ, using a novel MEMS-based heater technology provided by Protochips Co. (Raleigh, NC).

In-situ heating of the precursor materials provided insights into the diffusion processes of molecules and atomic clusters, and how the PtSn nanoparticles evolved. Figure 1a shows a high-angle annular dark-field (HAADF) image of the molecular precursor material, clearly revealing individual metalcontaining atoms and clusters dispersed onto the surfaces of the carbon black powders. Figure 2 shows an image of the same sample after in-situ reduction at a nominal $500^{\circ} \mathrm{C}$ for about 90 minutes, revealing the formation of ordered alloy nanoparticles as well as disordered nanoclusters. Figure $3 \mathrm{a}$ shows an image of the same sample after heating at a nominal $850^{\circ} \mathrm{C}$ for about 30 minutes, showing facets and the arrangement of surface atoms; the $4 \mathrm{~nm} \mathrm{PtSn}$ alloy nanoparticle consists primarily of $\{111\}$ facets; note the special atomic arrangement of the corner sites. The image also reveals the presence of many individual atoms and clusters on the surfaces of the carbon black (Fig. 3b). Detailed structural evolution of the nanoclusters with reduction temperature has been investigated. The utilization of pre-formed and compositionally well-defined bimetallic/multimetallic molecules as catalyst precursors provides a novel route to develop alloy nanoparticle catalysts, while in-situ aberration-corrected electron microscopy provides insights into the atomic-scale nucleation, diffusion and formation processes of metal and alloy nanoparticles [5].

References

[1] P Tsiakaras, Power Sources 171 (2007) 107.

[2] W Zhou et al., Appl. Catal. B: Environ. 46 (2003) 273.

[3] F Colmati, E Antolini and E. Gonzalez, Appl. Catal. B: Environ. 73 (2007) 106.

[4] C P White et al., Organometallics 26 (2007) 1996

[5] This research at the Oak Ridge National Laboratory's High Temperature Materials Laboratory was sponsored by the U. S. Department of Energy, Office of Energy Efficiency and Renewable Energy, Vehicle Technologies Program, and by the University of Missouri. 


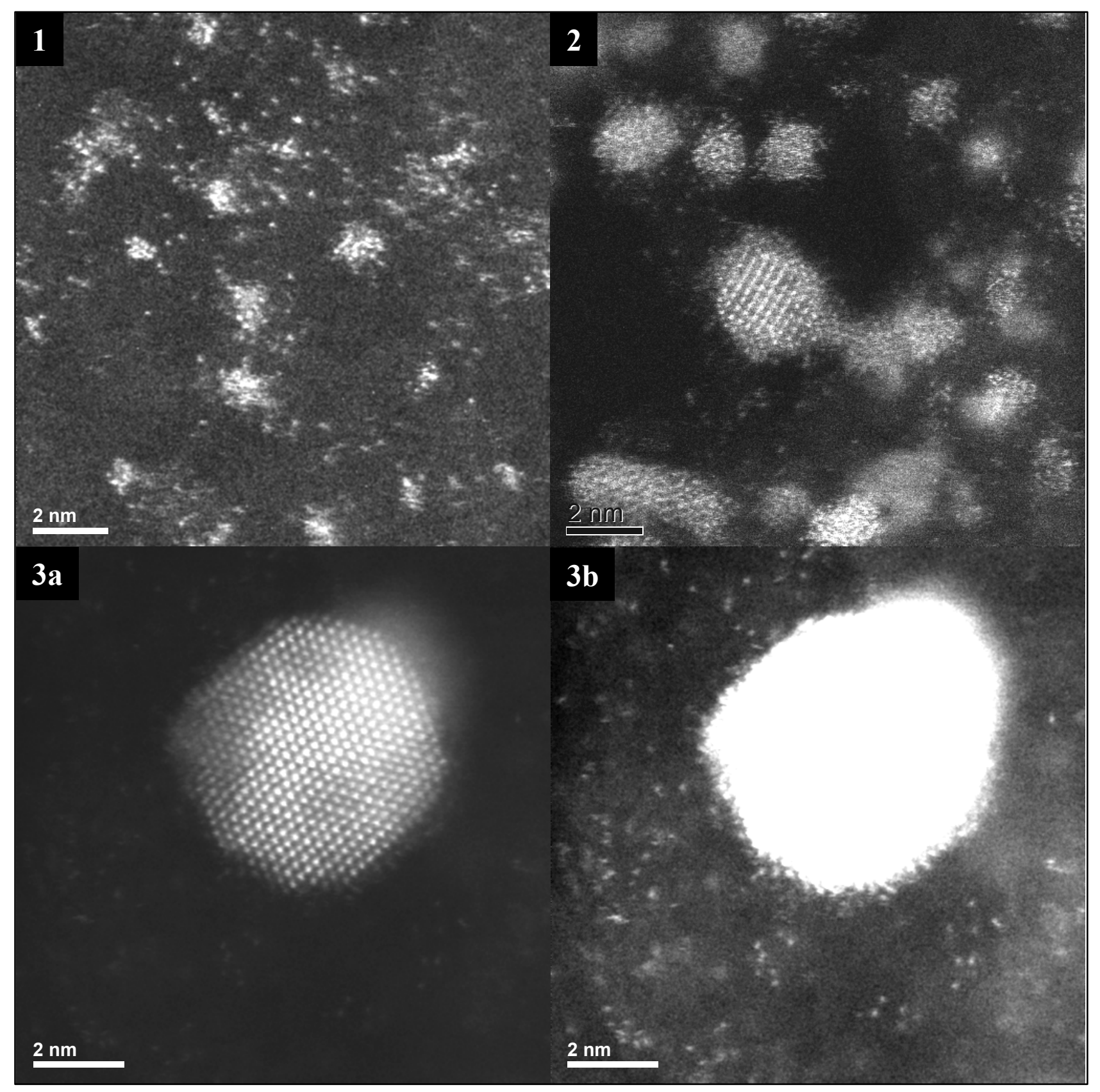

Fig. 1. Atomic resolution HA-ADF image of the $\mathrm{Pt}_{2} \mathrm{Sn}_{2}$ molecular precursors dispersed onto the surfaces of the XC-72 carbon black. Individual monomers and clusters are clearly resolved.

Fig. 2. Atomic resolution HA-ADF image of the $\mathrm{Pt}_{2} \mathrm{Sn}_{2} / \mathrm{C}$ molecular precursor material after being heated inside the microscope at nominal $500^{\circ} \mathrm{C}$ for 90 minutes, revealing individual atoms, disordered clusters and the formation PtSn alloy nanoparticles.

Fig. 3. Atomic-resolution HA-ADF image of the $\mathrm{Pt}_{2} \mathrm{Sn}_{2} / \mathrm{C}$ molecular precursor material after being heated inside the microscope at nominal $850^{\circ} \mathrm{C}$ for 30 minutes, revealing the formation of ordered alloy nanoparticles (a) and individual atoms and clusters near the alloy nanoparticle and on the carbon surface (b). Fig. 3b is the same image of Fig. 3a but displayed with increased brightness in order to show the atoms and clusters. 